\title{
ACKR1 Gene
}

National Cancer Institute

\section{Source}

National Cancer Institute. ACKR1 Gene. NCI Thesaurus. Code C78151.

This gene is involved in cytokine recognition. 